\title{
EXAMPLES OF ENTIRE FUNCTIONS WITH PATHOLOGICAL DYNAMICS
}

\author{
A. E. EREMENKO AND M. JU. LJUBICH
}

\section{Introduction}

The basic results of the iteration theory of rational and entire functions used in this paper are contained in the classical papers $[10,11]$, in the survey [5] and in $[\mathbf{1 5}$, Appendix III]. Let $f$ be a rational or entire function and let $f^{n}=f \circ \ldots \circ f$ be its $n$th iterate. Denote by $\mathscr{N}(f)$ the set of normality of $f$, that is, the maximal open set on which the family of iterates is normal in the sense of Montel. The complement of $\mathscr{N}(f)$ is called the Julia set $\mathscr{J}(f)$. The Julia set is non-empty, perfect and completely invariant, that is, it coincides with its inverse image.

A domain $\mathscr{D}$ is said to be wandering if $f^{m} \mathscr{D} \cap f^{n} \mathscr{D}=\varnothing$ for all $m>n \geqslant 0$. D. Sullivan [19] proved that the set of normality of a rational function has no wandering components. On the other hand I. N. Baker [3] constructed an entire function $f$ such that $\mathscr{N}(f)$ has a wandering component. This component $\mathscr{D}$ in Baker's example is multiply connected, $f^{m} \mathscr{D} \rightarrow \infty$ and $\operatorname{deg}\left(f^{m} \mid \mathscr{D}\right) \rightarrow \infty$ as $m \rightarrow \infty$. We construct several entire functions which have wandering domains with quite different properties.

EXAMPLE 1. There is an entire function $f$ which has a wandering component $\mathscr{D}$ of the set of normality such that every orbit $\left\{f^{n} z\right\}_{n=o}^{\infty}$ originating from $\mathscr{D}$ has an infinite limit set.

In this example all limit functions of the family $\left\{f^{n}\right\}$ in $\mathscr{D}$ are constants and the set of these constants is infinite.

EXAMPLE 2. There is an entire function $f$ which has a simply connected wandering component of $\mathcal{N}(f)$ in which all iterates $f^{n}$ are univalent.

Note that $M$. Herman [14] has also given an example of a simply connected wandering domain $\mathscr{D}$, but in his example $f^{n} \mathscr{D} \rightarrow \infty$ and $\operatorname{deg}\left(f^{n} \mid \mathscr{D}\right) \rightarrow \infty$ as $n \rightarrow \infty$.

The following example deals with the dynamics of an entire function $f$ in the invariant component of $\mathscr{N}(f)$ (a domain $\mathscr{D}$ is called invariant if $f \mathscr{D} \subset \mathscr{D}$ ). It is known that there are the following two types of dynamics in such a component.

(i) There exists a point $a \in \overline{\mathbb{C}}$ such that $f^{n} z \rightarrow a$ as $n \rightarrow \infty$ uniformly on the compact subsets of $\mathscr{D}$. If $f$ is rational, then $f a=a$. If $f$ is transcendental, then $f a=a$ or $a=\infty$.

(ii) The map $f: \mathscr{D} \rightarrow \mathscr{D}$ is conformally conjugate with an irrational rotation of a disk or a ring. For entire functions the case of a ring is impossible.

One of Fatou's main results states that in case (i) the rational function necessarily has a critical point in $\mathscr{D}$. In particular, $f$ is not univalent in $\mathscr{D}$. A similar fact is valid

Received 9 July 1986; revised 10 February 1987.

1980 Mathematics Subject Classification 30D05.

J. London Math. Soc. (2) 36 (1987) 458-468 
for transcendental functions: if $f^{n} z \rightarrow a \neq \infty$ in an invariant component $\mathscr{D}$ of the set of normality then $f: \mathscr{D} \rightarrow \mathscr{D}$ cannot be a covering map [2]. The following example shows that this is not true in the case when $a=\infty$.

EXAMPLE 3. There is an entire function $f$ which has an invariant component $\mathscr{D}$ such that $f^{n} z \rightarrow \infty$ as $n \rightarrow \infty, z \in \mathscr{D}$ and $f$ is univalent in $\mathscr{D}$.

Examples 1 to 3 are pathological from the point of view of the iteration theory of rational functions. Let us note, however, that there exists a rather wide class $S$ of entire functions having dynamical properties similar to those of rational functions. Define $S$ to be the class of functions $f$ such that there exists a finite set $A$, depending on $f$, such that $f: \mathbb{C} \backslash f^{-1} A \rightarrow \mathbb{C} \backslash A$ is a non-ramified covering map. It was established in $[6,7]$ that a function $f \in S$ has no wandering components of $\mathcal{N}(f)$. We recently learned that this result was independently obtained by L. Goldberg and L. Keen [13]. In [4] I. N. Baker proved the absence of wandering domains for a class of entire functions which is contained in $S$. Every orbit in $\mathcal{N}(f), f \in S$, is bounded $[6,7]$.

It is well known that the following alternative holds: the set $\mathcal{N}(f)$ is either dense or empty. A natural question arises therefore: is it true that in the first case the Julia set $\mathscr{J}(f)$ has Lebesgue measure zero? An affirmative answer to this question for rational $f$ would lead to a description of the dynamics of a generic rational function. For entire functions the answer is negative.

EXAMPLE 4. There is an entire function $f$ such that the Julia set $\mathscr{J}(f)$ is nowhere dense but mes $\mathscr{J}(f)>0$.

In the theory of quasiconformal deformations and structural stability of analytic dynamical systems $[8,9,16,17,19]$ the following important question arises: does there exist an invariant field of straight lines in $\mathscr{J}(f)$ ? We show that such a field does exist in Example 4. Slightly modifying this example we obtain the following.

EXAMPLE 5. There is an entire function $f$ which has an infinite-dimensional family of measurable invariant fields of straight lines in $\mathscr{J}(f)$.

The construction of our Examples 1 to 5 is based on the theory of approximation by entire functions. The necessary results from this theory are contained in $\$ 2$. Examples 1 to 3 are constructed in $\S 3$ and Examples 4 and 5 are in $\S 4$. The results of the paper were announced in [6], their detailed exposition in Russian was given in the preprint [7].

\section{Results on approximation by entire functions}

We shall make use of the following classical result.

RUNGE'S THEOREM. Let $K \subset \mathbb{C}$ be compact with connected complement. Then any function analytic on $K$ can be uniformly approximated by polynomials. (By a function analytic on $K$ we mean a function analytic in a neighbourhood of $K$.)

Using this theorem we prove a result on simultaneous approximation and interpolation. 
MAIN LEMMA. Let $\left\{G_{k}\right\}_{k-1}^{\infty}$ be a sequence of compact subsets of $\mathbb{C}$ with the following properties:

(i) $\mathbb{C} \backslash G_{k}$ is connected for every $k$;

(ii) $G_{k} \cap G_{m}=\varnothing$ for $k \neq m$;

(iii) $\min \left\{|z|: z \in G_{k}\right\} \rightarrow \infty$.

Let $z_{k} \in G_{k}, \varepsilon_{k}>0$ and the function $\phi$ be analytic on $G=\bigcup_{k \geqslant 1} G_{k}$. Then there exists an entire function $f$ satisfying

$$
\begin{gathered}
|f(z)-\phi(z)|<\varepsilon_{k}, \quad z \in G_{k} ; \\
f\left(z_{k}\right)=\phi\left(z_{k}\right), \quad f^{\prime}\left(z_{k}\right)=\phi^{\prime}\left(z_{k}\right), \quad k \in \mathbb{N} .
\end{gathered}
$$

The original proof of the main lemma due to the authors was considerably simplified by Ju. I. Ljubich who suggested that we apply the following geometrical statement.

LEMMA 1. Let $A$ be a locally convex linear topological space, let $V$ be a domain in $A$, let $W$ be a convex dense subset in $V$ and let $S$ be an affine subspace of $A$ of finite codimension, such that $S \cap V \neq \varnothing$. Then $S \cap W$ is dense in $S \cap V$.

Proof. By induction on codimension the proof is reduced to the case of $S$ having real codimension 1. Then $S$ is defined by the equation $F(g)=\mathscr{C}$ where $F$ is a continuous linear functional. Let $g \in V \cap S$ and let $B$ be a convex neighbourhood of $g$. Since $W$ is dense in $V$, we can find $f_{1}, f_{2} \in W \cap B$ such that $F\left(f_{1}\right)>\mathscr{C}, F\left(f_{2}\right)<\mathscr{C}$. Set $f=t f_{1}+(1-t) f_{2}$, where

$$
t=\frac{\mathscr{C}-F\left(f_{2}\right)}{F\left(f_{1}\right)-F\left(f_{2}\right)}
$$

We have $f \in W \cap B$ and $F(f)=\mathscr{C}$. Lemma 1 follows.

Proof of the main lemma. Let $\mathscr{U}$ be a simply connected neighbourhood of $G_{1}$ such that $\phi$ is analytic in $\mathscr{U}$ and $\mathscr{U} \cap G_{k}=\varnothing$ for $k \geqslant 2$. Consider the space $\mathscr{A}$ of all functions analytic in $\mathscr{U}$ with the topolgy of uniform convergence on compact sets. Consider the convex domain in $A$ :

$$
V=\left\{g:|g(z)-\phi(z)|<\frac{1}{2} \varepsilon_{1}, z \in G_{1}\right\} .
$$

Let $W$ be the subset of $V$ consisting of polynomials. By Runge's theorem $W$ is dense in $V$. Clearly $W$ is also convex. Now consider the affine subspace

$$
S=\left\{g \in A: g\left(z_{1}\right)=\phi\left(z_{1}\right), g^{\prime}\left(z_{1}\right)=\phi^{\prime}\left(z_{1}\right)\right\} .
$$

By Lemma 1 there exists $f_{1} \in W \cap S$; that is, $f_{1}$ is a polynomial satisfying

$$
\begin{gathered}
\left|f_{1}(z)-\phi(z)\right|<\frac{1}{2} \varepsilon_{1}, \quad z \in G_{1}, \\
F_{1}\left(z_{1}\right)=\phi\left(z_{1}\right), \quad f_{1}^{\prime}\left(z_{1}\right)=\phi^{\prime}\left(z_{1}\right) .
\end{gathered}
$$


By a similar argument we find a sequence of polynomials $f_{m}$ with the following properties

$$
\begin{gathered}
\left|\phi(z)-\sum_{k=1}^{m} f_{k}(z)\right|<\frac{1}{2} \varepsilon_{m}, \quad z \in G_{m} ; \\
\left|f_{m}(z)\right|<2^{-m+k} \varepsilon_{k}, \quad z \in G_{k}, k<m ; \\
\left|f_{m}(z)\right|<2^{-m}, \quad|z|<\frac{1}{2} \min \left\{|\zeta|: \zeta \in G_{m}\right\} ; \\
\sum_{k=1}^{m} f_{k}\left(z_{i}\right)=\phi\left(z_{i}\right), \quad \sum_{k=1}^{m} f_{k}^{\prime}\left(z_{i}\right)=\phi^{\prime}\left(z_{i}\right), \quad 1 \leqslant i \leqslant m .
\end{gathered}
$$

It follows from (5) that the series $f=\sum_{m=1}^{\infty} f_{m}$ converges uniformly on the compact subsets of $\mathbb{C}$ and defines an entire function. Finally (3), (4) imply (1) and (6) implies (2). The main lemma is proved.

Furthermore, we need two technical lemmas.

LEMMA 2. Let $f(z)=z+g(z)$ be an analytic function in the disk $\{z:|z|<R\}$ such that $g(0)=g^{\prime}(0)=0$ and $|g(z)|<\varepsilon R$ for $|z|<R$ and some $\varepsilon<\frac{1}{2}$. Then

$$
\begin{aligned}
& |z|\left(1-\frac{\varepsilon}{R}|z|\right) \leqslant|f(z)| \leqslant|z|\left(1+\frac{\varepsilon}{R}|z|\right) ; \\
& |\arg f(z)-\arg z|<2 \frac{\varepsilon}{R}|z|, \quad|z|<R .
\end{aligned}
$$

Proof. The function $g(z) / z^{2}$ is analytic in $\{z:|z|<R\}$ and by the maximum principle its modulus does not exceed $\varepsilon / R$. This immediately implies (7). Furthermore,

$$
|\arg f(z)-\arg z| \leqslant|\log (f(z) / z)|=|\log (1+g(z) / z)| \leqslant 2|g(z) / z| \leqslant 2 \frac{\varepsilon}{R}|z|,
$$

and $(8)$ is also proved.

LEMma 3. Let $q>1$. Then there exists a number $s(q)$ such that the estimates

$$
r_{0} \sum_{k=0}^{m-1} \varepsilon_{k}<s(q), \quad r_{0}>0, \varepsilon_{k}>0
$$

and

imply that

$$
r_{k}\left(1-\varepsilon_{k} r_{k}\right) \leqslant r_{k+1} \leqslant r_{k}\left(1+\varepsilon_{k} r_{k}\right), \quad 0 \leqslant k \leqslant m-1
$$

$$
\frac{1}{q} r_{0} \leqslant r_{k} \leqslant q r_{0}, \quad 0 \leqslant k \leqslant m .
$$

Proof. Let

$$
r_{0} \sum_{k=0}^{m-1} \varepsilon_{k}<\frac{1}{q} \log q
$$

Suppose inductively that $r_{i} \leqslant q r_{0}, 0 \leqslant i \leqslant k-1$. Then we obtain

$$
r_{k} \leqslant r_{0} \prod_{i=0}^{k-1}\left(1+\varepsilon_{i} r_{i}\right) \leqslant r_{0} \exp \left(\sum_{i=0}^{k-1} \varepsilon_{i} r_{i}\right) \leqslant r_{0} \exp \left(q r_{0} \sum_{i=0}^{k-1} \varepsilon_{i}\right)
$$

By (9) the last expression does not exceed $q r_{0}$. 
Now choose $\gamma>1$ such that the estimate $1-x \geqslant \exp (-\gamma x)$ holds for $0<x<\frac{1}{2}$. Suppose that

$$
r_{0} \sum_{k=0}^{m-1} \varepsilon_{k}<\min \left\{\frac{1}{q \gamma} \log q, \frac{1}{2 q}\right\}:=s(q) .
$$

We have already proved that under this condition the estimates $r_{i}<q r_{0}, 0 \leqslant i \leqslant m$ hold. Thus we have

$$
r_{k} \geqslant r_{0} \prod_{i=0}^{k-1}\left(1-\varepsilon_{i} r_{i}\right) \geqslant r_{0} \exp \left(-\gamma \sum_{i=0}^{k-1} \varepsilon_{i} r_{i}\right) \geqslant r_{0} \exp \left(-\gamma r_{0} q \sum_{i=0}^{k-1} \varepsilon_{i}\right) \geqslant r_{0} / q
$$

Lemma 3 is proved.

\section{Pathological dynamics on the set of normality}

EXAMPLE 1. There is an entire function $f$ having a wandering component $\mathscr{D}$ of the set of normality such that every orbit $\left\{f^{n} z\right\}_{n=0}^{\infty}$ originating from $\mathscr{D}$ has an infinite limit set.
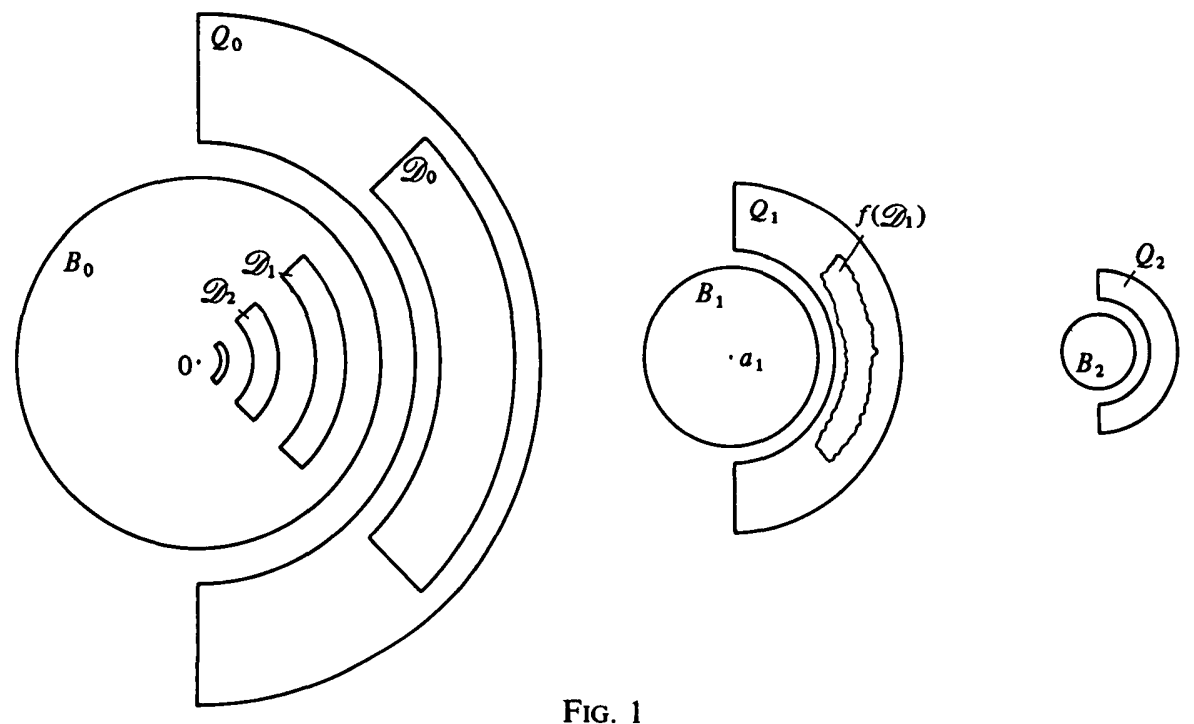

Fix $\varepsilon, q$ such that $0<\varepsilon<\frac{1}{2}, 1<q<2^{\frac{1}{3}}$, and consider a sequence $\left\{R_{m}\right\}_{m-0}^{\infty}$ satisfying

$$
\begin{gathered}
0<R_{m}<\frac{1}{2} R_{m-1}, \quad m=1,2, \ldots ; \\
\varepsilon \sum_{k=0}^{m-1} \frac{R_{m}}{R_{k}}<\min \left\{s, \frac{1}{8} \pi\right\}, \quad m=1,2, \ldots,
\end{gathered}
$$

where $s=s(q)$ is chosen by Lemma 3. Define a sequence $\left\{a_{m}\right\}_{m=0}^{\infty}$ such that $a_{0}=0$, $a_{m+1}-a_{m}>2 R_{0}$. Let

$$
\begin{gathered}
B_{m}=\left\{z:\left|z-a_{m}\right| \leqslant \frac{1}{2} R_{m}\right\}, \\
\mathscr{D}_{m}=\left\{z: q^{-2} R_{m}<|z|<q^{-1} R_{m},|\arg z|<\frac{1}{4} \pi\right\}, \\
Q_{m}=\left\{z: q^{-3} R_{m}<\left|z-a_{m}\right|<R_{m},|\arg z|<\frac{1}{2} \pi\right\}
\end{gathered}
$$


(see Figure 1), and define the function $\phi$ on the set $\bigcup_{m=0}^{\infty}\left(B_{m} \cup Q_{m}\right)$ in the following way:

$$
\begin{gathered}
\phi(z)=z+a_{m+1}-a_{m}, \quad z \in B_{m}, m \in \mathbb{Z}_{+} ; \\
\phi(z)=q^{-\frac{3}{2}} R_{m+1}, \quad z \in Q_{m}, m \in \mathbb{Z}_{+} .
\end{gathered}
$$

By the main lemma there exists an entire function $f$ satisfying

$$
\begin{gathered}
f\left(a_{m}\right)=a_{m+1}, \quad f^{\prime}\left(a_{m}\right)=1, \quad m \in \mathbb{Z}_{+} ; \\
|f(z)-\phi(z)|<\frac{1}{2} \varepsilon R_{m}, \quad z \in B_{m}, m \in \mathbb{Z}_{+} ; \\
f\left(Q_{m}\right) \subset \mathscr{D}_{m+1}, \quad m \in \mathbb{Z}_{+} .
\end{gathered}
$$

We show that $\mathscr{D}_{0} \subset \mathcal{N}(f)$ and the iterates $f^{n} D_{0}$ have constant limit functions $a_{m}$.

Let $|z|<\frac{1}{2} R_{m}, r_{k}=\left|f^{k} z-a_{k}\right|, k \in \mathbb{Z}_{+}$. We shall prove that

$$
q^{-1} r_{0} \leqslant r_{k} \leqslant q r_{0}, \quad 0 \leqslant k \leqslant m .
$$

Suppose inductively that

$$
q^{-1} r_{0} \leqslant r_{i} \leqslant q r_{0}, \quad 0 \leqslant i \leqslant k-1<m .
$$

As $q r_{0}<\left(2^{\frac{1}{3}} / 2\right) R_{m}<R_{m}<\frac{1}{2} R_{i}$, it follows that $f^{i} z \in B_{i}, 0 \leqslant i \leqslant k-1$. Then, from (12) and (13) and Lemma 2 , we have

By (11),

$$
r_{i}\left(1-\varepsilon \frac{r_{i}}{R_{i}}\right) \leqslant r_{i+1} \leqslant r_{i}\left(1+\varepsilon \frac{r_{i}}{R_{i}}\right), \quad 0 \leqslant i \leqslant k-1 .
$$

Using Lemma 3 we obtain

$$
r_{o} \sum_{i=0}^{m-1} \frac{\varepsilon}{R_{i}} \leqslant \varepsilon \sum_{i=0}^{m-1} \frac{R_{m}}{R_{i}}<s
$$

$$
q^{-1} r_{0} \leqslant r_{k} \leqslant q r_{0} .
$$

Thus (15) is proved by induction, and we have

$$
f^{k} z \in B_{k}, \quad 0 \leqslant k \leqslant m-1 .
$$

Using Lemma 2 once more, we obtain

$$
\left|\arg \left(f^{k+1} z-a_{k+1}\right)-\arg \left(f^{k} z-a_{k}\right)\right| \leqslant 2 \varepsilon \frac{r_{k}}{R_{k}} \leqslant 2 \varepsilon \frac{R_{m}}{R_{k}}, \quad 0 \leqslant k \leqslant m-1 .
$$

Therefore, by (11),

$$
\left|\arg \left(f^{m} z-a_{m}\right)-\arg z\right| \leqslant 2 \varepsilon \sum_{k=0}^{m-1} \frac{R_{m}}{R_{k}}<\frac{\pi}{4}
$$

It follows from this and (15) (for $k=m$ ) that $f^{m} \mathscr{D}_{m} \subset Q_{m}, m \in \mathbb{Z}_{+}$. Since $f Q_{m} \subset \mathscr{D}_{m+1}$, by (14), it follows that $a_{0}=0$ is a limit point for every orbit originating from $\mathscr{D}_{0}$. Hence all points $a_{m}$ have the same property. The required example is constructed.

Problem. Does there exist an entire function $f$ and a component $\mathscr{D}$ of $\mathcal{N}(f)$ such that the limit functions of subsequences of $f^{n}$ in $\mathscr{D}$ form an infinite bounded set?

To construct Examples 2 and 3 we need the following approximation theorem (see for example [12]). 
ARAKElJAN's TheOREM. Let $G \subset \mathbb{C}$ be a closed set. The following properties are equivalent:

(i) every function $\phi$ continuous on $G$ and analytic in the interior of $G$ can be uniformly approximated by entire functions;

(ii) $\overline{\mathbb{C}} \backslash G$ is connected and locally connected at $\infty$.

EXAMPLE 2. There is an entire function $f$ having a simply connected wandering component of $\mathcal{N}(f)$ in which all iterates are univalent. Let us consider the following vertical strips symmetric with respect to the lines $\{z: \operatorname{Re} z=3 m\}, m=10,11, \ldots$ :

$$
\begin{gathered}
\Pi_{m}=\{z:|\operatorname{Re} z-3 m|<1\}, \\
\Pi_{m}^{\prime}=\left\{z:|\operatorname{Re} z-3 m|<1-2^{-m-2}\right\}, \\
\Pi_{m}^{\prime \prime}=\left\{z:|\operatorname{Re} z-3 m|<1-2^{-m}\right\} .
\end{gathered}
$$

Let $\phi_{m}$ be a linear function which maps $\Pi_{m}^{\prime \prime}$ onto $\Pi_{m+1}$. Obviously

$$
\left|\operatorname{Re}\left(\phi_{m}(z)-3(m+1)\right)-\operatorname{Re}(z-3 m)\right|<2^{-m}, \quad z \in \Pi_{m}^{\prime \prime} .
$$

Now consider the strip

$$
\Pi^{*}=\left\{z:|\operatorname{Re} z-30|<\frac{1}{2}\right\}
$$

It follows from (16) that

$$
\phi_{m} \circ \phi_{m-1} \circ \ldots \circ \phi_{10} \Pi^{*} \subset \Pi_{m+1}^{\prime \prime}, \quad m=10,11, \ldots
$$

Using Arakeljan's theorem construct the function $f$ satisfying

$$
\left.\begin{array}{l}
\left|f(z)-\phi_{m}(z)\right|<2^{-3 m}, \quad z \in \Pi_{m}^{\prime} ; \\
|f(z)|<1, \quad z \in \partial \Pi_{m}, m=10,11, \ldots ; \\
|f(z)|<1, \quad z \in \mathbb{U}=\{z:|z|<1\} .
\end{array}\right\}
$$

The unit disk is invariant and hence it is contained in $\mathscr{N}(f)$. Further, it is easy to see that $f^{m} \Pi^{*} \subset \Pi_{m+10}^{\prime \prime}$. Consequently $\Pi^{*}$ is contained in $\mathcal{N}(f)$. Let $\mathscr{D}$ be the component of $\mathcal{N}(f)$ containing $\Pi^{*}$. Then $f^{m} z \rightarrow \infty$ in $\mathscr{D}$. However if $z \in \partial \Pi_{m}$ then by (17) the orbit $\left\{f^{m} z\right\}_{m-0}^{\infty}$ is bounded. Thus $f^{m} \mathscr{D} \in \Pi_{m+10}$ and $\mathscr{D}$ is a wandering component.

Now we check that the iterates of $f$ are univalent in $\mathscr{D}$. Consider the strips

$$
\mathscr{L}_{m}=\left\{z:|\operatorname{Re} z-3 m|<1-2^{-m-1}\right\} .
$$

It is easy to see that $f \mathscr{L}_{m} \supset \Pi_{m+1}$ and hence that $f^{m} \mathscr{D} \subset \mathscr{L}_{m+10}$. Let us show that $f$ is univalent in $\mathscr{L}_{m}$. The Cauchy formula for derivatives implies that

$$
f(z)=z+3+g(z), \quad\left|g^{\prime}(z)\right|<\frac{1}{2}
$$

for $z \in \mathscr{L}_{m}$. Consequently, if $f\left(z_{1}\right)=f\left(z_{2}\right) ; z_{1}, z_{2} \in \mathscr{L}_{m}$, then

$$
\left.\left|z_{1}-z_{2}\right|=\mid g\left(z_{1}\right)-g z_{2}\right)\left|\leqslant \frac{1}{2}\right| z_{1}-z_{2} \mid
$$

and hence $z_{1}=z_{2}$. Thus univalence is proved.

To check that $\mathscr{D}$ is simply connected we apply the following lemma (see [4, Theorem 3.1, Corollary] or [7, Proposition 3]). 
LEMMA 4. Let f be an entire function bounded on a curve tending to $\infty$. Then all the components of $\mathcal{N}(f)$ are simply connected.

This lemma completes the investigation of Example 2.

EXAmple 3. There is an entire function $f$ which has an invariant component $\mathscr{D}$ of the set $\mathcal{N}(f)$ such that $f^{n} z \rightarrow \infty$ as $n \rightarrow \infty, z \in \mathscr{D}$, and $f$ is univalent in $\mathscr{D}$.

Let us consider the half-planes

$$
P_{1}=\{z: \operatorname{Re} z>-4\}, \quad P_{2}=\{z: \operatorname{Re} z<-5\} .
$$

Define the functions $f_{1}(z)=2 z$ in $P_{1}$ and $f_{2}(z)=\exp z-6$ in $P_{2}$. By Arakeljan's theorem there exists an entire function $f$ such that

$$
\left|f(z)-f_{i}(z)\right|<\frac{1}{2}, \quad z \in P_{i}, i=1,2 .
$$

This implies that $\operatorname{Re} f(z)>\operatorname{Re} z+\frac{1}{2}$ for $z \in \mathscr{D}_{0}=\{z: \operatorname{Re} z>1\}$. Hence all orbits in $\mathscr{D}_{0}$ tend to $\infty$. Furthermore we have

$$
\sup \left\{|f(z)+6|: z \in P_{2}\right\}<1 .
$$

Hence all orbits in $P_{2}$ tend to an attractive fixed point. Thus $\mathscr{D}_{0}$ and $P_{2}$ are contained in different components of $\mathcal{N}(f)$. Let $\mathscr{D}$ be the component of $\mathcal{N}(f)$ containing $\mathscr{D}_{0}$. Consider the line $\mathscr{L}=\{z: \operatorname{Re} z=-3\} \subset P_{1}$. We have $f \mathscr{L} \subset P_{2}$ and hence

$$
\mathscr{D} \subset \mathscr{Q}=\{z: \operatorname{Re} z>-3\} \text {. }
$$

Let us show that $f$ is univalent in 2 . Let $f(z)=2 z+g(z)$. Then $|g(z)|<\frac{1}{2}$ in each disk of radius 1 centred at $z \in \mathscr{Q}$. Hence $\left|g^{\prime}(z)\right|<\frac{1}{2}, z \in \mathscr{Q}$. If $f\left(z_{1}\right)=f\left(z_{2}\right) ; z_{1}, z_{2} \in \mathscr{Q}$, then

$$
2\left|z_{1}-z_{2}\right|=\left|g\left(z_{1}\right)-g\left(z_{2}\right)\right| \leqslant \frac{1}{2}\left|z_{1}-z_{2}\right|,
$$

which implies that $z_{1}=z_{2}$. Thus $f$ is univalent in the half-plane $\mathscr{Q}$ and, moreover, in $\mathscr{D}$.

RemarK. M. Herman [15] gave an elementary example with the same properties as those in Example 3.

\section{Julia sets of positive area}

EXAMPLE 4. There is an entire function $f$ such that the Julia set $\mathscr{J}(f)$ is nowhere dense but mes $\mathscr{J}(f)>0$.

Consider a sequence $\varepsilon_{k}>0$ such that

and the sequences of squares

$$
36 \sum_{k=0}^{\infty} \varepsilon_{k}<1,
$$

$$
\begin{gathered}
Q_{k}=\{x+i y:|x-4 k|<1,|y|<1\}, \\
Q_{k}^{1}=\left\{x+i y:|x-4 k|<1+\varepsilon_{k},|y|<1+\varepsilon_{k}\right\}, \quad k=0,1, \ldots .
\end{gathered}
$$

Delete the following two strips from each $Q_{k}$ :

$$
\mathscr{L}_{k}=\left\{x+i y: x-4 k \mid<\varepsilon_{k}\right\}, \quad \mathscr{M}_{k}=\left\{x+i y:|y|<\varepsilon_{k}\right\} .
$$

The set $Q_{4} \backslash\left(\mathscr{L}_{k} \cup \mathscr{M}_{k}\right)$ is the union of four squares $Q_{k, j}, 1 \leqslant j \leqslant 4$. Consider the affine surjective map $\phi_{k, j}: Q_{k, j} \rightarrow Q_{k+1}^{1}$. 
Using the main lemma (or Arakeljan's theorem) construct an entire function $f$ satisfying

(i) $\left|f(z)-\phi_{k, j}(z)\right|<\varepsilon_{k+1}, z \in Q_{k, j}$;

(ii) $f$ is univalent in $Q_{k, j}$ and $\left|f^{\prime}(z)\right|>2, z \in Q_{k, j}$;

(iii) $f$ has an attractive fixed point $\alpha=-2$.

To verify condition (ii) we may apply the same reasoning as in Examples 2 and 3. It follows from (iii) that $\mathscr{J}(f)$ is nowhere dense. We have

where

$$
Q_{k+1} \subset f Q_{k, j} \subset Q_{k+1}^{\prime \prime},
$$

$$
Q_{k+1}^{\prime \prime}=\left\{x+i y:|x-4(k+1)|<1+2 \varepsilon_{k+1},|y|<1+2 \varepsilon_{k+1}\right\} .
$$

Consider the set

$$
K^{(n)}=\left\{z: f^{k} z \in \bigcup_{j=1}^{4} \bar{Q}_{k, j}, k=0,1, \ldots, n\right\} .
$$

It is the union of $4^{n+1}$ disjoint closed sets $K_{i_{0}, i_{1}, \ldots, i_{n}}^{(n)}, 1 \leqslant i_{k} \leqslant 4$ on each of which $f^{n}$ is univalent. Since diam $K_{i_{0}, i_{1}, \ldots, i_{n}}^{(n)} \leqslant 2^{-n}$ it follows that $K^{(\infty)}=\bigcap_{n=0}^{\infty} K^{(n)}$ is a Cantor set.

We show that mes $K^{(\infty)}>0$. Denote $Q_{n}^{\prime \prime} \backslash \bigcup_{j-1}^{4} Q_{n, j}$ by $E_{n}$. We have mes $E_{n} \leqslant 36 \varepsilon_{n}$. As $\left|\left(f^{n}\right)^{\prime}\right|>2^{n}, z \in K^{(n)}$, it follows that

$$
\operatorname{mes}\left(K_{i_{0}, \ldots, i_{n}}^{(n)} \cap f^{-n-1} E_{n+1}\right) \leqslant 36 \varepsilon_{n+1} \cdot 4^{-n-1} \text {. }
$$

Hence $\operatorname{mes}\left(K^{(n)} \cap f^{-n-1} E_{n+1}\right) \leqslant 36 \varepsilon_{n+1}$. As $K^{(n+1)}=K^{(n)} \backslash f^{-n-1} E_{n+1}$, the required statement follows from (18).

Furthermore if $z \in K^{(\infty)}$ then $\left|f^{n}(z)\right|=O(n), n \rightarrow \infty$, and $\left|\left(f^{n}\right)^{\prime}(z)\right|>2^{n}$. Thus the spherical derivative

$$
\left|\left(f^{n}\right)^{\prime}(z)\right| /\left(1+\left|f^{n}(z)\right|^{2}\right) \rightarrow \infty, \quad n \rightarrow \infty, z \in K^{(\infty)},
$$

and so $K^{(\infty)} \subset \mathscr{J}(f)$.

Remark. In Example 4 as constructed,

$$
\operatorname{mes}\left\{z \in \mathscr{J}(f):\left|f^{m} z\right| \rightarrow \infty, m \rightarrow \infty\right\}>0 .
$$

Combining the ideas of this example and of Example 1 we may construct an entire function $f$ for which

$$
\operatorname{mes}\left\{z \in \mathscr{J}(f): \varliminf_{n \rightarrow \infty}\left|f^{n} z\right|<\infty\right\}>0 .
$$

Problem. Construct an entire function $f$ with the property that

$$
\operatorname{mes}\left\{z \in \mathscr{J}(f): \sup _{n}\left|f^{n} z\right|<\infty\right\}>0 .
$$

EXAMPLE 5. There is an entire function $f$ having an infinite-dimensional family of measurable invariant fields of straight lines on $\mathscr{J}(f)$.

Given a field of straight lines on a set $X \subset \mathbb{C}$, let $\theta(z)$ be the angle between the line corresponding to $z \in X$ and the positive ray of the real axis. As $\theta(z)$ is defined only modulo $\pi z$ it is convenient to determine the field of lines by the function

$$
\mu(z)= \begin{cases}\exp 2 i \theta(z), & z \in X, \\ 0, & z \in \mathbb{C} \backslash X .\end{cases}
$$


A field is called measurable if the function $\mu$ is measurable. Such a field is defined almost everywhere and is considered as non-trivial if mes $X>0$. We mean that $\mu$ defines the field not only on $X$ but on all sets $Y \supset X$. The field is called invariant (under $f$ ) if

$$
\mu(f x)=\left(f^{\prime}(x) /\left|f^{\prime}(x)\right|\right)^{2} \mu(x) .
$$

We show that in Example 4 there exists a non-trivial invariant field of lines on $\mathscr{J}(f)$. Set $c_{k}=\phi_{k, j}^{\prime}(z)>2, z \in Q_{k, j}$. Let $\delta_{k}>0, \sum_{k=0}^{\infty} \delta_{k}<\infty$ and the approximation of $\phi_{k, j}$ by $f$ is so close that $\left|f^{\prime}(z)-c_{k}\right|<\delta_{k}, z \in Q_{k, j}$. Then

$$
\left.\left|\arg f^{\prime}(z)\right|=\mid \operatorname{Im} \log \left(f^{\prime}(z)\right) / c_{k}\right)|\leqslant A| f^{\prime}(z) / c_{k}-1 \mid \leqslant A \delta_{k}, \quad z \in Q_{k, j}
$$

Thus the series $\theta(z)=-\sum_{k=0}^{\infty} \arg f^{\prime}\left(f^{k} z\right)$ converges absolutely and uniformly on the set $\mathscr{L}=\bigcup_{n=0}^{\infty} f^{n} K^{(\infty)}$ and defines there a continuous function. Moreover,

$$
\theta(f z)=\theta(z)+\arg f^{\prime}(z)
$$

and consequently $\theta$ defines a non-trivial invariant field of lines on $\mathscr{L}$. This field has the natural extension to an invariant field on the completely invariant set

$$
\bigcup_{n=0}^{\infty} f^{-n} \mathscr{L}=\mathscr{L}^{*}
$$

To construct the entire function required in Example 5, one has to carry out the construction of Example 4 simultaneously on an infinite set of sequences of squares. One obtains infinitely many disjoint completely invariant sets $\mathscr{L}_{k}^{*}$ having positive measure. Moreover, there exists an invariant field of lines $\mu_{k}$ on each $\mathscr{L}_{k}^{*}$. These fields generate an infinite-dimensional family of invariant fields $\sum_{k=1}^{\infty} \lambda_{k} \mu_{k}, \lambda_{k} \in \mathbb{C},\left|\lambda_{k}\right|=1$.

REMARK C. McMullen [18] proved that mes $\mathscr{J}(f)>0$ for $f(z)=\sin z$. This Julia set cannot carry an infinite-dimensional family of invariant fields of lines because $f \in S$.

\section{References}

1. I. N. BAKER, 'Repulsive fixpoints of entire functions', Math. Z. 104 (1968) 252-256.

2. I. N. BAKER, 'Limit functions and sets of non-normality in iteration theory', Ann. Acad. Sci. Fenn. (Ser. AI) 467 (190) 1-11.

3. I. N. BAKER, 'An entire function which has wandering domains', J. Austral. Math. Soc. (Ser. A) 22 (1976) 173-176.

4. I. N. BAKER, 'Wandering domains in the iteration of entire functions', Proc. London Math. Soc. (3) 49 (1984) 563-576.

5. P. Blanchard, 'Complex analytic dynamics on the Riemann sphere', Bull. Amer. Math. Soc. 11 (1984) 85-141.

6. A. E. Eremenko and M. Ju. Luubich, 'Iterates of entire functions', Soviet Math. Dokl. 30 (1984) 592-594.

7. A. E. Eremenko and M. Ju. LJubich, 'Iterates of entire functions', preprint, Physico-Technical Institute of Low Temperatures Ukrain SSR Academy of Sciences, Kharkov, N 6 (1984) 1-37.

8. A. E. ERemenko and M. Ju. LJUbich, 'Structural stability in some families of entire functions', preprint, Physico-Technical Institute of Low Temperatures Ukrain SSR Academy of Sciences, Kharkov, N 29 (1984) 1-36.

9. A. E. Eremenko and M. JU. LJubich, 'Structural stability in some families of entire functions', Funkt. Anal. Appl. 19 (1985) 86-87.

10. P. Fatou, 'Sur les équations fonctionnelles', Bull. Soc. Math. France 47 (1919) 161-271; 48 (1920) 33-94; 208-314.

11. P. Fatou, 'Sur l'itération des fonctions transcendantes entières', Acta Math. 47 (1926) 337-370.

12. D. GaIER, Vorlesungen über Approximation im Komplexen (Birkhäuser, Basel, 1980).

13. L. Goldberg and L. KeEN, 'A finiteness theorem for a dynamical class of entire functions', Ergod. Theory Dynam. Systems 6 (1986) 183-192. 
14. M. HeRman, 'Exemples de fractions rationnelles ayant une orbite dense sur la sphère de Riemann', Bull. Soc. Math. France 112 (1984) 93-142.

15. M. Herman, 'Are there critical points on the boundaries of singular domains?', Commun. Math. Phys. 99 (1985) 593-612.

16. M. Ju. LuUbiCH, 'Some generic dynamical properties of rational mappings', Uspehi Mat. Nauk 38 (1983) 197-198.

17. R. Mané, P. SAD and D. Sullivan, 'On the dynamics of rational maps', Ann. Sci. Ecole Norm. Sup. 16 (1983) 193-217.

18. C. MCMullen, 'Area and Hausdorff dimension of Julia sets of entire functions', preprint, MSRI, Berkeley, California, 1986, 1-15.

19. D. Sullivan, 'Itération des fonctions analytiques complexes', C. R. Acad. Sci. 294 (1982) 301304.

Institute for Low Temperature Physics and Engineering Academy of Sciences Ukrain SSR

47 Lenin Prospect

310164 Kharkov

USSR 